\title{
Quantum Entanglement and the Emergence of Collaboration in Social Media
}

\author{
Nazaria Solferino* \\ Department of Economics and Finance \\ University of Calabria, Ponte Pietro Bucci \\ Cubo O/C, 87036 Arcavacata di Rende, CS, Italy \\ nazaria.solferino@uniroma2.it \\ Viviana Solferino \\ Department of Mathematics and Computer Science \\ University of Calabria \\ 87036 Arcavacata di Rende, CS, Italy \\ Serena Fiona Taurino \\ Department of Economics and Law, Unitelma Sapienza \\ University of Rome \\ Viale Regina Elena 295, 00161 Rome, Italy
}

Received 12 May 2018

Accepted 29 June 2018

Published 18 July 2018

\begin{abstract}
In this paper, we devise a slightly modified version of the vote with the wallet game used by Becchetti et al. [L. Becchetti and F. Salustri, The vote with the wallet as a multiplayer prisoner's dilemma, CEIS Tor Vergata Research Paper No. 359, Vol. 13, Issue 10, Centre for Economic and International Studies, Rome, Italy (2015); L. Becchetti, V. Pelligra and F. Salustri, Testing for heterogeneity of preferences in randomized experiments: A satisfactionbased approach applied to multiple prisoner dilemmas, Appl. Econ. Lett. 24(10) (2017) 722726] for the use of social media, where the player decides whether to responsibly share social knowledge or not. We follow the point of view of Bennet and Bennet [D. Bennet and A. Bennet, Social learning from the inside out: The creation and sharing of knowledge from the mind/brain perspective, in Social Knowledge: Using Social Media to Know What You Know, eds. J. P. Girard and J. L. Girard (IGI Global, 2011), pp. 1-23] according to which another social settings may emerge through the so-called "process of collaborative entanglement." In this environment, members of a community interact continuously with strong emotional feelings to combine the sources of knowledge and the beneficiaries of that knowledge and move toward a common direction. The application of the model to the quantum game theory
\end{abstract}

\footnotetext{
${ }^{*}$ Corresponding author.
}

This is an Open Access article published by World Scientific Publishing Company. It is distributed under the terms of the Creative Commons Attribution 4.0 (CC-BY) License. Further distribution of this work is permitted, provided the original work is properly cited. 
substantially confirms that the cooperative strategy becomes the optimal one depending on the frequency of interactions and people's cultural, geographical and social reachability and traceability.

Keywords: Quantum games; cooperative games; social media.

JEL Classification: C71, D03, Z10.

\section{Introduction}

Thanks to the Internet and the digital technologies, nowadays we can share information and knowledge, spread culture and education faster and more easily than in the past. The collective body of knowledge produced by the community or social circle is what is known as social knowledge. In a social or cultural context, social knowledge can be the collective knowledge based on small groups, like a family, or it can be a massive and constantly evolving body of knowledge, like Wikipedia. According to the theory of social knowledge it is not simply the total sum of a group's knowledge, but it is the very product of relationships and connections within a particular group. These groups are known as social knowledge networks, and their size and influence can often depend on the group topics, features and relationships. On a much larger scale, the Internet has produced massive social knowledge networks through websites or other sites where people regularly gather to share information on a particular topic. Websites like these are known as wiki, namely a digital application that allows for collaboration and often requires user-generated content to remain relevant. The social knowledge networks and wikis do not have a control system for the verification of the information, but they are an evolving collective knowledge resource produced by community participation. Therefore, the most critical challenge is how to use them effectively in a collaborative way as well as to avoid the fake news. There is a broad range of literature which analyzes the problem of how to detect fake news, trolling and haters (see, e.g., Refs. 16 and 17). Besides that, there is also the rising phenomenon of online incivility, which has made the environment of social networking sites hostile to many users. In a recent work, Antoci et al. ${ }^{1}$ studied how the effect of social media on trust varies depending on the civility or incivility of online interactions. Thanks to an experiment on Facebook, the authors found that participants exposed to "civic-minded" Facebook interactions are significantly more trusting (for a survey on this topic see the literature cited in Ref. 1). In this paper, we explore another way of stimulating cooperation on the use of social media, based on the model of collaborative entanglement. ${ }^{4}$ This is a highly participatory model where knowledge creators, who may be individual, team or community, and the audience interact continuously with strong emotional feeling to combine the sources of knowledge and the beneficiaries of that knowledge. Acting so, they move toward a common direction to satisfy community needs. From these interactions emerge both a new knowledge formed by learned expertise and a large amount of tacit knowledge (embodied, effective and intuitive) due to the continuing 
iterative loop of collective learning, that visibly affects the community. Therefore, "the process of collaborative entanglement among individuals not only helps to provide a specific solution to a current issue, but seeds the ground for continuous community self improvement, collaboration and sustainability." ${ }^{4}$ We analyze this framework with the use of a quantum game model, substantially based on the pioneering work of Eisert and Wilkens. ${ }^{8}$ Quantum game models have been largely applied in game theory mainly to show how new cooperative equilibria arise when players act as entangled particles. In their recent work, Guo et al. ${ }^{11}$ depict a complete survey of these models and their several applications. In this paper, our aim is to stress the importance of media education, namely the education for the fair and correct use of social media since the school age, also with the collaboration of families. Our starting point is that, given the level of dissemination the use of social media has reached, they have become of essential use nowadays. However, the existence of many haters and fake news can make the use of social media dangerous for the sensible dissemination of knowledge and, even worst, can harm people. Benvenuto et $a l .{ }^{5}$ provide a systematic large-scale measurement and analysis study of hate speech in online social media. In their work, they use large datasets from two social media providers: Whisper and Twitter. The first dataset counts up to more than 27 million whispers and the second gathers more than 512 million tweets. Furthermore, the authors set up and validate a methodology to identify hate speech and delineate a definition of hate speech. According to them, hate speech is "an offensive post, motivated, in whole or in a part, by the writer's bias against an aspect of a group of people. The offended aspects can encompass offline hate crimes, based on race, religion, disability, sexual orientation, ethnicity, or gender. However, they might also include behavioral and physical aspects." In light of the above, besides the positive spillovers of knowledge dissemination, the use of social media brings out the need of protection from haters as well as from fake news. To protect ourselves, we can use appropriate methods and laws, but they can be very expensive and not always feasible. Although enforcement methods and law are fundamental, the entanglement approach represents another additional (not alternative) solution which consists in educating people, since the school age, for an appropriate use of social media. According to this approach, the right information benefits everyone not only because it increases social knowledge, but also because people feel better as they do their civic duty, belong to a group, increase their own awareness and the awareness of others, not to mention the positive spillovers of correct information shared among individuals. We see this through a simple model of game theory, by using a version of the modified game of Becchetti et al., ${ }^{2,3}$ which is more suitable since our purpose is a cooperative game model in which the change comes from citizens, from bottom-up pressure. In Sec. 2, we devise this modified version by highlighting the importance of laws and methods to correct fake news and haters. While in Sec. 3, we see the additional effects of the entanglement model; by applying our model to the quantum game theory, we substantially confirm that the cooperative strategy becomes the optimal one depending on frequency of interactions and people's cultural, 
geographical and social reachability and traceability. In Sec. 4, we discuss the media education importance to enforce the entanglement in practice. In Sec. 5, we summarize our model and the main results.

\section{The Basic Model}

In this section, we aim to set up the basic features and parameters that we think are at work in a classical game when the agents must decide whether to cooperate or not in building and sharing social knowledge. To this purpose, we extensively draw from the model of Becchetti et $a .^{2,3}$ by using a slightly modified version of that game where now we denote by:

- $c$ the real costs (bills, expenses to stay connected, etc.) and the cost opportunity of the free time spent on social media instead of other leisure activities or face-to-face interactions.

- $b$ the positive benefit related to the enrichment in knowledge due to the exchange of information.

- $a$ the individual advantage derived from interacting with other people at a distance or just from releasing emotions and contents.

- $h$ the number of haters.

The individual payoff associated to the strategy of cooperation is

$$
P_{C C}=b\left(1-\frac{f}{2}\right)+a\left(l-e \frac{h}{2}\right)-c,
$$

where $f$ is the cost to detect fake news, which we may assume is proportional to the amount of the exchanged information (i.e., the more news and websites people see, the more probably they can incur fake news). The parameter $e$ measures the number of haters that we assume are exogenous. That is, we adopt the point of view of hating as psychological disordered behavior which is substantially independent of the others' responses and which worsens individual well-being proportionally to individual feelings of empathy and sensitivity measured by the variable $e$. Finally, the parameter $l$ measures how much $a$ increases thanks to the others' reply, for instance, with the number of "likes" and emoticons or comments of approval received. Similarly, the payoff the agent gets when the strategy chosen is to cooperate while the other one defects is

$$
P_{C D}=\frac{b}{2}(1-f)+\frac{a}{2}(l-e h)-c,
$$

where now the agent must support the entire cost of detecting fake news $f$. The defector instead gets $P_{D C}=\frac{b}{2}+\frac{a}{2}$. If both players choose the strategy of defecting they both get 0 . All the parameters are clearly non-negative: $a, b, c, f, h$, $l, e \in[0, \infty]$. 
Therefore the final set of payoffs may be written as follows:

$$
U_{i}(S)= \begin{cases}b\left(1-\frac{f}{2}\right)+a\left(l-e \frac{h}{2}\right)-c & \text { if } S=(C, C), \\ \frac{b}{2}(1-f)+\frac{a}{2}(l-e h)-c & \text { if } S=(C, D), \\ \frac{b}{2}+\frac{a}{2} & \text { if } S=(D, C), \\ 0 & \text { if } S=(D, D) .\end{cases}
$$

The Nash equilibrium (NE) of this social knowledge responsible game (hereinafter $\mathrm{SKR})$ is the cooperative solution $(C, C)$ if $\frac{b}{2}\left(1-\frac{f}{2}\right)+\frac{a}{2}(l-e h)>c$, while defection prevails otherwise. Therefore having a cooperative equilibrium in this classical game strongly depends on the following:

(i) The costs of detecting fake news $f$ : The seriousness of the problem of the raising fake news and their misconception damages makes critical the need to develop new techniques to tackle this challenge. To this end, Facebook has recently announced a series of efforts towards addressing this challenge. ${ }^{10}$ Fake news and misinformation have historically been used as tools for making political or business gains. ${ }^{9}$ The institutions have been proposing several solutions, as well as scientific literature is full of examples and propositions. One of the most interesting can be the detection of fake news via expert's verification or fact-checking (see, e.g., Ref. 14). This methodology has given rise to some third-party fact-checking organizations such as Snopes3 and Factcheck.org 4 and the code of principles ${ }^{12}$ that should be followed by these organizations is built up. However, the volume of news content that is generated in online social networks has become so vast and the speed of spread in these networks is so fast that developing new computational techniques and complementing the expert verification are strongly required (see, for instance, Ref. 6, for a survey). An alternate approach is to build hybrid human-algorithm methods via engaging users of online social networks by enabling them to report the fake news. ${ }^{16}$

(ii) The number of haters $h$ : Hate speech is expressly considered in the laws of many countries that define it as "a speech intended to degrade, intimidate or incite violence or prejudicial action against someone based on her race, ethnicity, national origin, religion, sexual orientation, or disability." When coming to statutory debarment of the hate speech, controversies arise. First, because of the conflict with the shielded right to freedom of expression of every citizen. However, one can counter-argument that the violence of some hate speeches, the ways they depict marginalized individuals or groups sometimes reach a demeaning and derogatory pick so that freedom of 
expression is quickly put out of weight. As it was not enough, we have to add that marginalized people do need societal protection against any form of abuse. In our society, interlacing mechanisms of oppressions make hate speech even powerful and dangerous to the very zoa (i.e., "natural life") of individuals, not only to their bios politikos (i.e., "the good life characteristic of participation in the polis"). It is indeed the case that hate speech not only can humiliate, chastise and exclude the person or the group they hurl abuse at, but will harshly damage them by discriminating them according to gender, race, sexuality, disability status, religion, etc. This risk of harming people and going back to old — but not soothe - discrimination culture is perhaps the primary reason why more and more countries are adopting laws against hate speech. For instance, very exemplary is the case of Germany which has recently enforced a law that demands social media sites move quickly to remove hate speech, fake news and illegal materials. Websites that do not delete "obviously illegal" posts could face fines of up to 50 million euros. The law gives the networks $24 \mathrm{~h}$ after notice to act against the law-breaking material.

\section{The Model of Quantum Entanglement in Social Media}

The quantum entanglement is a physical phenomenon that occurs when pairs or groups of particles interact in a way such that, even when they are separated by a large distance, the quantum state of each particle cannot be described independently of the others. That is, they are not individual particles but are an inseparable whole. To better understand the property of entanglement we can assume the two particles are located in two distant countries $A$ and $B$. If we want to measure a particular characteristic of one of these particles, after we get a result then measure the other particle using the same criterion and we find that the result of the measurement of the second particle will match (in a complementary sense) the result of the measurement of the first particle.

The quantum mechanics and the concept of entanglement have been largely applied in recent years in game theory, shedding light on new important results with respect to the traditional ones. For instance, in their pioneering work on quantum games Eisert and Wilkins ${ }^{8}$ investigated the quantization of non-zero sum games. For the particular case of the prisoners' dilemma, they show that it is ever possible to construct a particular quantum strategy which always gives a reward if played against any classical strategy.

In this paper, we strongly refer to this quantum version of the prisoner's dilemma as developed in Ref. 8 and we apply it to a context of our social knowledge responsible game devised in the previous section. We aim to show that, depending on the players' sociocultural and social proximity and interactions, the cooperative quantum strategy may give a higher reward with respect to the classical one even when the number of haters and the costs of fake news make it not profitable. 
We rewrite the classical game in terms of matrix as

$$
\begin{aligned}
& \left(\begin{array}{ccc} 
& C & D \\
C & (r, r) & (p, t) \\
D & (t, p) & (s, s)
\end{array}\right) \\
& \begin{array}{|c|c|c|}
\hline & C & D \\
\hline & b\left(1-\frac{f}{2}\right)+a\left(1-e \frac{h}{2}\right) & b \\
C & -c, b\left(1-\frac{f}{2}\right)+a\left(1-e \frac{h}{2}\right)-c & \frac{b}{2}(1-f)+\frac{a}{2}(1-e h)-c, \frac{a}{2}+\frac{b}{2} \\
\hline D & \frac{a}{2}+\frac{b}{2}, \frac{b}{2}(1-f)+\frac{a}{2}(1-e h)-c & (0,0) \\
\hline
\end{array}
\end{aligned}
$$

where we have normalized $l$ to 1 .

For a quantum formulation of the classical game of SRK developed in Sec. 2, we proceed like Eisert and Wilkins ${ }^{8}$ by assigning the possible outcomes of the classical strategies $C$ and $D$ two basis vectors

$$
|C\rangle=\left(\begin{array}{l}
1 \\
0
\end{array}\right), \quad|D\rangle=\left(\begin{array}{l}
0 \\
1
\end{array}\right)
$$

in the Hilbert space of a two-state system, i.e., a qubit. At each instance, the state of the game is described by a vector in the tensor product space which is spanned by the classical game bases $|C C\rangle,|C D\rangle,|D C\rangle$ and $|D D\rangle$, where the first and second entries refer to the first and the second players, called $A$ and $B$, respectively. The board of our quantum-game is depicted in Fig. 1.

The final total expected payoff may be derived as the weighted sum of each possible payoff and may be in general written as (for more details about how to derive a quantum game see Ref. 8):

$$
\Pi=r P_{C C}+p P_{C D}+t P_{D C}+s P_{D D} .
$$

Therefore, the payoff operators for each of the two players are

$$
\begin{aligned}
& P_{A}\left(S_{A}, S_{B}\right)=\left[b\left(1-\frac{f}{2}\right)+a\left(1-e \frac{h}{2}\right)-c\right] P_{C C}+0 \cdot P_{D D}+\left(\frac{a}{2}+\frac{b}{2}\right) P_{D C} \\
& +\left[\frac{b}{2}(1-f)+\frac{a}{2}(1-e h)-c\right] P_{C D}
\end{aligned}
$$

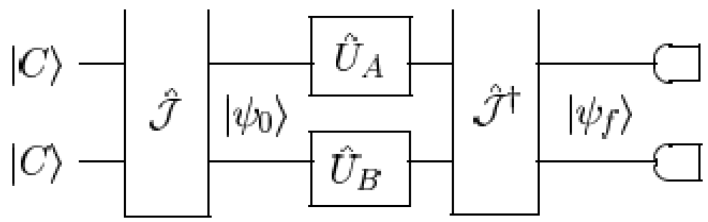

Fig. 1. The setup of a two-player quantum game. 


$$
\begin{aligned}
P_{B}\left(S_{A}, S_{B}\right)= & {\left[b\left(1-\frac{f}{2}\right)+a\left(1-e \frac{h}{2}\right)-c\right] P_{C C}+0 \cdot P_{D D}+\left(\frac{a}{2}+\frac{b}{2}\right) P_{C D} } \\
& +\left[\frac{b}{2}(1-f)+\frac{a}{2}(1-e h)-c\right] P_{D C} .
\end{aligned}
$$

We denote the game's initial state by $\left|\Psi_{0}\right\rangle=\hat{J}|C C\rangle$ where $\hat{J}$ is the entanglement operator acting on the two bits. Then final state of the quantum game is

$$
\left|\Psi_{f}\right\rangle=\hat{J}^{\dagger}\left(\hat{U}_{A} \otimes \hat{U}_{B}\right) \hat{J}|C C\rangle
$$

where $\hat{J}^{\dagger}$, its adjoint, is the disentanglement operator, and $\hat{U}_{A}, \hat{U}_{B}$ are the single-bit operators selected by the two players, that in other words measure the density matrix applied to the expected payoff to evaluate the final result.

Specifically, $\hat{J}$ is the $4 \times 4$ matrix

$$
\hat{J}=\frac{1}{\sqrt{2}}\left(I+\sigma_{x} \otimes \sigma_{y}\right)=\frac{1}{\sqrt{2}}\left(\begin{array}{cccc}
1 & 0 & 0 & i \\
0 & 1 & i & 0 \\
0 & i & 1 & 0 \\
i & 0 & 0 & 1
\end{array}\right),
$$

where $\sigma_{x}$ is the $2 \times 2$ Pauli matrix $\left(\begin{array}{ll}0 & 1 \\ 1 & 0\end{array}\right)$. The general single-qubit operator or density matrix which must be applied to the expected payoff is

$$
\left(\begin{array}{cc}
e^{i \phi} \cos \frac{\theta}{2} & \sin \frac{\theta}{2} \\
-\sin \frac{\theta}{2} & e^{-i \phi} \cos \frac{\theta}{2}
\end{array}\right)
$$

where $0 \leq \theta \leq \pi$ and $0 \leq \phi \leq \frac{\pi}{2}$.

The angle $\theta$ and the spin $\phi$ represent the analogs of players' sociocultural and social proximity and frequency of interactions as well as the possibility of opening up, recognizability, reachability, e.g., when the particles have spin $\phi=0$ it means that players are maximally distant or can never meet (as when particles are on parallel lines). Therefore for $A$ and $B$ we have, respectively:

$$
\begin{aligned}
& \hat{U}_{A}=\hat{U}\left(\theta_{A}, \phi_{A}\right)=\left(\begin{array}{cc}
e^{i \phi_{A}} \cos \frac{\theta_{A}}{2} & \sin \frac{\theta_{A}}{2} \\
-\sin \frac{\theta_{A}}{2} & e^{-i \phi_{A}} \cos \frac{\theta_{A}}{2}
\end{array}\right), \\
& \hat{U}_{B}=\hat{U}\left(\theta_{B}, \phi_{B}\right)=\left(\begin{array}{cc}
e^{i \phi_{B}} \cos \frac{\theta_{B}}{2} & \sin \frac{\theta_{B}}{2} \\
-\sin \frac{\theta_{B}}{2} & e^{-i \phi_{B}} \cos \frac{\theta_{B}}{2}
\end{array}\right) .
\end{aligned}
$$


Given the choices $\theta_{A}, \phi_{A}$ and $\theta_{B}, \phi_{B}$ of the players, matrix multiplication gives the following resulting probabilities:

$$
\begin{aligned}
P_{C C} & =\cos ^{2}\left(\phi_{A}+\phi_{B}\right) \cos ^{2} \frac{\theta_{A}}{2} \cos ^{2} \frac{\theta_{B}}{2}, \\
P_{C D} & =\left[\sin \phi_{B} \sin \frac{\theta_{A}}{2} \cos \frac{\theta_{B}}{2}-\cos \phi_{A} \cos \frac{\theta_{A}}{2} \sin \frac{\theta_{B}}{2}\right]^{2}, \\
P_{D C} & =\left[\sin \phi_{A} \cos \frac{\theta_{A}}{2} \sin \frac{\theta_{B}}{2}-\cos \phi_{B} \sin \frac{\theta_{A}}{2} \cos \frac{\theta_{B}}{2}\right]^{2}, \\
P_{D D} & =\left[\sin \frac{\theta_{A}}{2} \sin \frac{\theta_{B}}{2}+\sin \left(\phi_{A}+\phi_{B}\right) \cos \frac{\theta_{A}}{2} \cos \frac{\theta_{B}}{2}\right]^{2} .
\end{aligned}
$$

We can get the final payoffs for the agents $A$ and $B$ by Eqs. (5) and (6).

The equilibrium solution shows that a lower threshold now is requested to make more convenient, for both players, to benefit from deviation from $D$. The cooperative strategy is associated to the couple $(\theta, \phi)=\left(0, \frac{\pi}{2}\right)$, while the defection strategy corresponds to $(\theta, \phi)=(\pi, 0)$. In particular, another Nash equilibrium emerges when each player chooses the cooperative strategy $(\theta, \phi)=\left(0, \frac{\pi}{2}\right)$, as the associated individual payoff now is equal to $P_{C C}$ which is higher than $P_{D D}$. This Nash equilibrium, that we call $(\hat{Q}, \hat{Q})$, is unique and serves as the only acceptable solution of the game. No player could gain without lessening the other player's expected payoff. In this sense one can say that the dilemma of the original game has fully disappeared.

If a quantum game is not maximally entangled, i.e., the probabilities of the possible outcomes of the game are not uniformly distributed, the entanglement operator may be rewritten as follows (see also Ref. 15):

$$
\hat{J}=e^{i \frac{\gamma}{2} \hat{D} \otimes \hat{D}}
$$

where the parameter $\gamma \in\left[0, \frac{\pi}{2}\right)$ can be considered as a measure for the game's entanglement that allows to determine the entropy

$$
E=\sin ^{2} \frac{\gamma}{2} \ln \sin ^{2} \frac{\gamma}{2}-\cos ^{2} \frac{\gamma}{2} \ln \cos ^{2} \frac{\gamma}{2}
$$

of the initial state $\left|\Psi_{0}\right\rangle$.

For a maximally entangled quantum game $\gamma=\frac{\pi}{2}$ and the unique feasible NE of the quantum game is $(\hat{Q}, \hat{Q})$. Otherwise, when the game is not maximally entangled we adopt $\hat{Q}$ only as cooperator's strategy and the modified matrix in the non-maximally entangled quantum game becomes

$$
\left(\begin{array}{cc}
b\left(1-\frac{f}{2}\right)+a\left(1-e \frac{h}{2}\right)-c & \left(\frac{a}{2}+\frac{b}{2}\right) \sin ^{2} \gamma \\
\left(\frac{a}{2}+\frac{b}{2}\right) \cos ^{2} \gamma & \frac{b}{2}(1-f)+\frac{a}{2}(1-e h)-c
\end{array}\right)
$$


The prisoner's dilemma is resolved if

$$
\sin \gamma>\frac{b}{2}(1-f)+\frac{a}{2}(1-e h)-c,
$$

which is ever satisfied for $\gamma \in\left[0, \frac{\pi}{2}\right)$.

For simplicity we restrict our attention to the production and benefits of social knowledge, so that we assume $a=0, c=0$. Notice that while in the classical version

of the game for $\frac{b}{2}\left(1-\frac{f}{2}\right)<0$, i.e., $f>2$, the cooperative strategy never represents a feasible NE, instead in the quantum game it is a possible solution depending only on (9).

Therefore, the above condition for a cooperative unique NE substantially means that the benefit that comes from interactions entangled with each other even when defecting is a greater for the player than when he chooses to cooperate while others are defecting but without entanglement. This result substantially depends on the values of the parameters $\theta, \phi$ and $\gamma$. Notice that in our analysis essentially $\theta$ and $\phi$ represent the distance and frequency of interactions among agents. Therefore, the entanglement is greater for less distant people (not only geographically but also in terms of cultural and social differences) and when they are easily reachable/traceable. In this case, even if the costs of detecting fake news (and fighting haters) are huge, the entanglement gives to the agents additional benefits when cooperating that derive from contributing to the change in the society, in spreading the right information, as well as rising awareness, self-esteem, altruism, fairness, developing critical thinking, etc. This makes the cooperative strategy more attractive also when the other agents might defect if the entropy of the system is not too high, i.e., $\gamma \in\left[0, \frac{\pi}{2}\right]$.

This result has been confirmed by the recent paper of Sabatini and Sarracino ${ }^{13}$ who show that the use of social networks has a positive impact on well-being also because it increases the probability of face-to-face interactions.

\section{Discussion}

Nowadays, social media are part of our daily culture and communicative practices in the family, at school and with the group of friends. In this paper, we have devised a simple quantum game theoretical model on how to foster a more beneficial and fair use of social media. This model is based on the physical concept of entanglement. In this section we discuss how it may be built in the society through the work of schools and families. First of all, it is necessary to understand the basic consumer practices and values inside the educational system (i.e., schools) so as to create awareness among the students about the use of social media. On the other hand, schools should promote training meetings to involve families to make them more attentive to their children's media consumption both in terms of content and emotions. Besides that, the media have to be considered as an area to explore as well as to challenge. The problem of media education was also expressively taken into account by the 
European Parliament, through the resolution of 16 December, 2008. The Parliament recognized the importance of media education as an essential element to increase cultural literacy, awareness and to promote the active civic participation of people. Consequently, the Parliament alleged that media education should be part of formal education to which all children have access and are an integral part of the educational program in all kinds of schools and degrees.

An exemplary case in this regard is represented in Italy by the schools involved in the Italian project of Corecom (http://www.corecomragazziemiliaromagna.it). The project was carried out for five years during which, several activities have been realized. The mainstream consisted in the analysis of online news or info, the realization of written texts aimed at online publications. The purpose of the project is to try to understand the explicit as well as the implied meaning. They play both like journalists, writers and readers so that they will learn all the techniques to understand articles, opinions and all the other forms of text shared via social media. In other words, they carry out role simulations with the aim of learning how to interpret all the info shared via social media. This methodology has been substantially inspired by the five principles of pedagogy of media education identified by De Smedt et al. ${ }^{7}$

The first, used very frequently by teachers, consists in the practical production of media, i.e., it requires students to create a transmission radio, an announcement, a short film, a publication or a text. Putting students in the position of producers is considered by educators a relevant way for students to evolve their relationship with the media. The second is the decomposition of message and consists in choosing a medium and analyzing it deeply to discover which mechanisms of significance are at work, what elements are contained and how they produce meaning. The third pedagogy consists in teaching directly some concepts, through frontal lessons, to learn some contents taken, for example, from the Humanities (economics, anthropology, sociology, political science), which are very useful to analyze the media. The fourth is based on introspective processes: it invites the pupil to take awareness of his own perceptions, of the different modifications that the media provoke on his sensibility, his needs, feelings, concerns, expectations in an environment of sharing and socializing of experiences and opinions, using specific and appropriate terms. Finally, the fifth pedagogy De Smedt et al. ${ }^{7}$ suggested refers to the game and problem solving: simulation games, roles and activities that do not propose the only media production, but that present to the young students a problem that needs to be solved. Pupils have to find strategies by themselves; the solution they will find will be a new competence that they will have acquired.

The aim of social education is to increase media literacy and acquire the key competence in the information and communication society. So that all potential users get the ability to autonomously use the various media, to understand and evaluate the different aspects of content of media, to communicate in heterogeneous contexts and to produce and disseminate media content, and to filter and classify the information sought in the tide of data and images offered by the new media. Media education is a key element of consumer information policy, a conscious and 
competent approach to copyright issues, the active democratic participation of citizens and the promotion of intercultural dialog. This is necessary to finally return to the media their primary function of information dissemination in real time and to break down the inability to communicate at a distance instead of being transformed as it happens nowadays as a tool of opportunistic falsification of news or a basket to regurgitate one's own negative emotions.

\section{Conclusions}

The proliferation of haters and fake news is becoming a very relevant problem nowadays. It involves each kind of subject from politics and elections to celebrities' life, chronicles, history, etc., at a such point that it has elicited alarm from institutions, scientific community and individuals. Alongside the phenomenon of haters, it poisons civic dialog, threatens the social cohesion and democracy. In this paper we address this problem from a theoretical point of view by using an innovative approach based on quantum game theory findings. Out starting point is how to foster social media to get back their role of place for reciprocal beneficial sharing of social knowledge to increase common well-being? To this aim we use a slightly modified version of the vote with the wallet game by Becchetti et $a .^{2,3}$ to define the main variables that are at work and how they affect final payoffs and equilibrium of the game. According to our version of the model, clearly fake news and the number of haters reduce the benefits associated to sharing social knowledge, depending on individual's empathy, costs of detecting fake news, social approval, etc. In Sec. 2, we discussed the most traditional means to deal with the problems related to how to detect fake news, mainly by devising special algorithms that intermediaries may use for fact-checking and the regulations needed to prevent the proliferation of haters. In Sec. 3, strongly inspired from the work of Bennet and Bennet, ${ }^{4}$ we try to suggest an additional approach based on education about how to use social media and develop what in physics is called entanglement. It is a type of empathic cohesion arising between entities interacting also at very distant locations. This is just the case of people using social media who can interact in real time, from different locations and at different points of time. We apply our model to a simple quantum game analysis to show that when entanglement is at work, even when costs of detecting fake news and dealing with haters are high, sharing social knowledge may still be highly beneficial for all the agents involved. This strongly depends on the frequency of interactions and traceability and reachability of each agent. In Sec. 4, we discuss the relevance of schools and families which play the main roles to create an education system devoted to promote and spread entangled environments when kids start learning how to use social media.

\section{Acknowledgments}

The authors thank Prof. L. Becchetti for his useful suggestions about the basic idea of the paper. They also thank the music artist R. J. Radke for inspiring the idea with 
his vicissitudes and sayings, ever pushing for the search of the truth behind fake news and the wall of haters to make social media a place "not to tear someone down...but to enjoy seeing everyone wins."

\section{References}

1. A. Antoci, A. Delfino, F. Paglieri, F. Panebianco and F. Sabatini, Civility vs. incivility in online social interactions: An evolutionary approach, PLoS ONE 11(11) (2016) e0164286.

2. L. Becchetti, V. Pelligra and F. Salustri, Testing for heterogeneity of preferences in randomized experiments: A satisfaction-based approach applied to multiplayer prisoners' dilemmas, Appl. Econ. Lett. 24(10) (2017) 722-726.

3. L. Becchetti and F. Salustri, The vote with the wallet as a multiplayer prisoner's dilemma, CEIS Research Paper No. 359, CEIS, Tor Vergata University (2015).

4. D. Bennet and A. Bennet, Social learning from the inside out: The creation and sharing of knowledge from the mind/brain perspective, in Social Knowledge: Using Social Media to Know What You Know, eds. J. P. Girard and J. L. Girard (IGI Global, 2011), pp. 1-23.

5. F. Benevenuto, M. Mondal and L. A. Silva, A measurement study of hate speech in social media, in Proc. 28th ACM Conf. Hypertext and Social Media (2017).

6. N. J. Conroy et al., Automatic deception detection: Methods for finding fake news, Proc. Assoc. Inf. Sci. Technol. 52(1) (2015) 1-4.

7. T. De Smedt, J. Jerry and P. Fastrez, Organising media as social objects: An exploratory assessment of a core media literacy competence, Media Educ. Res. J. 40(1) (2013) 42-57.

8. J. Eisert and M. Wilkins, Quantum games, J. Mod. Opt. 47 (2000) 2543-2556.

9. S. Ewen, A Social History of Spin (Basic Books, 1998).

10. Facebook, News feed FYI: Addressing hoaxes and fake news (2016), https://newsroom. fb.com/news/2016/12/news-feed-fyi-addressing-hoaxes-and-fake-news/.

11. H. Guo, J. Zhang and G. J. Koehler, A survey of quantum games, Decis. Support Syst. 46(1) (2008) 318-332.

12. Poynter, International fact-checking network: Fact-checkers code principles (2016), https://www.poynter.org/internationalfact-checking-network-fact-checkerscode-principles.

13. F. Sabatini and F. Sarracino, Online networks and subjective well-being, Kyklos 70(3) (2017) 456-480.

14. N. Solferino, S. F. Taurino and M. E. Tessitore, Manipulating persuasion in debates: Fact checking's usefulness, Theor. Econ. Lett. 5(4) (2015) 447-452.

15. Z. W. Sun, The rule for evolution of cooperation in quantum games, Acta Phys. Pol. A 116(2) (2009) 135-140.

16. S. Tschiatschek, A. Singla, M. G. Rodriguez, A. Merchant and A. Krause, Fake news detection in social networks via crowd signals, arXiv:1711.09025v2 [cs.SI].

17. A. S. Figueira and L. Oliveira, The current state of fake news: Challenges and opportunities, Procedia Computer Science 121 (2017) 817-825. 